\title{
Peculiar nature of hard X-ray eclipse in SS433 from INTEGRAL observations
}

\author{
A. Cherepashchuk ${ }^{* a}$, K. Postnov ${ }^{a}$, E. Antokhina ${ }^{a}$, S. Molkov ${ }^{b}$ \\ ${ }^{a}$ Sternberg Astronomical Institute, 119992 Moscow, Russia \\ ${ }^{b}$ CESR, Toulouse, France \\ E-mail: cherepashchuk@gmail . com, kpostnov@gmail.com, \\ elant@sai.msu.ru, Sergey.Molkov@cesr.fr
}

\begin{abstract}
The analysis of hard X-ray INTEGRAL observations (2003-2008) of superaccreting galactic microquasar SS433 at precessional phases of the source with the maximum disk opening angle is carried out. It is found that the shape and width of the primary X-ray eclipse is strongly variable suggesting additional absorption of X-ray photons in dense stellar wind and gas outflows from the optical A7I-component. The joint modeling of X-ray eclipse and precessional X-ray variability of SS433 revealed by INTEGRAL using a geometrical model suggests the binary mass ratio $q=m_{x} / m_{v} \simeq 0.3$. This value of the binary mass ratio allows us to easily explain peculiarities of the optical variability of SS433, in particular, the substantial precessional variability of the minimum brightness at the middle of the primary optical eclipse. For the mass function of the optical star $f_{v}=0.268 M_{\odot}$ as derived from Hillwig \& Gies data [14] the obtained value of $q$ yields the masses of the components $m_{x} \simeq 5 M_{\odot}, m_{v} \simeq 15 M_{\odot}$, confirming the black hole nature of the relativistic object in SS433. The independence of the observed hard X-ray spectrum on the accretion disk precession phase suggests that hard X-ray emission $(k T=20-100 \mathrm{keV})$ is formed in an extended, hot, quasi-isothermal corona, probably heated by interaction of relativistic jet with inhomogeneous wind outflow from the precessing supercritical accretion disk. The Monte-Carlo simulations of broadband X-ray spectrum of SS433 at the maximum disk opening precessional phases allowed us to determine physical parameters of the hot corona (temperature $T_{\text {cor }}=20 \mathrm{keV}$, Thomson optical depth $\tau=0.2$ ), as well as to estimate the mass outflow rate in jets $\dot{M}_{j}=3 \times 10^{19} \mathrm{~g} / \mathrm{s}$ yielding the kinetic power of the jets $\sim 10^{39} \mathrm{erg} / \mathrm{s}$.
\end{abstract}

7th INTEGRAL Workshop

September 8-11 2008

Copenhagen, Denmark

\footnotetext{
* Speaker.
} 


\section{Introduction}

SS433 is a massive close binary system at advanced evolutionary stage $[17,5,10]$. This unique galactic X-ray binary with precessing relativistic jets $(v=0.26 c$, where $c$ is the speed of light) exhibits several variabilities, including the precessional one (with the period $P_{\text {prec }} \simeq 162 \mathrm{~d}$ ), the eclipsing one (with the binary orbital period $P_{\text {orb }}=13.08 \mathrm{~d}$ ), and the nutational one (with the nutation period $P_{n u t} \simeq 6.28 \mathrm{~d}$ ) (e.g. [13]). SS433 is recognized as a galactic microquasar with precessing supercritical accretion disk around a relativistic compact object, and has been extensively investigated in the optical, radio and X-ray ranges (for a comprehensive review and references see [10]). The optical spectroscopy of the system [14] revealed the presence of absorption lines in the spectrum of the optical component identified as a $\sim$ A7I supergiant star. Observed orbital Doppler shifts of the absorption lines of the optical component allowed Hillwig \& Gies to determine the mass ratio of the relativistic $\left(m_{x}\right)$ and the optical $\left(m_{v}\right)$ components in SS433 $q=m_{x} / m_{v} \simeq 0.35$, implying the masses $m_{x}=4.3 \pm 0.8 M_{\odot}$ and $m_{v}=12.3 \pm 3.3 M_{\odot}$. Similar masses were obtained from our analysis of the optical light curves of SS433 [2].

Until now, the analysis of X-ray eclipses in SS433 in the 1-10 keV range has yielded controversial results suggesting a small mass ratio $q \simeq 0.15$ (e.g. [15]). The main reason for this is a very broad X-ray eclipse observed in this X-ray band.

Our studies of SS433 in hard X-rays using the INTEGRAL observations suggested the presence of a hot rarefied corona above the supercritical accretion disk in this source [7, 8, 9]. The peculiar variability in the shape and width of the primary eclipse in hard X-rays was discovered. This implies that the primary eclipse is not purely geometrical and that the binary mass ratio as derived from the eclipse duration may be unrealistic.

The results of our analysis of the orbital and precessional variability in SS433 observed by INTEGRAL [9] can be summarized as follows. The hard X-ray flux (25-50 keV) from the source clearly exhibits the precessional variability with the precessional period $P_{\text {prec }}=162.4 \mathrm{~d}$ from $\sim$ $3 \mathrm{mCrab}$ at the cross-over phase to $\sim 18-20 \mathrm{mCrab}$ at the maximum disk opening phase (the $T_{3}$ moment, where the moving emission lines in the SS433 spectrum are at maximum separation). The orbital eclipses are observed with the orbital period $P_{\text {orb }}=13.08 \mathrm{~d}$ and are very significant. Eclipses observed close to the $T_{3}$ phase (at the precessional phase $\psi \simeq 0.1$ ) are the deepest ones. The hard $\mathrm{X}$-ray flux (18-60 keV) at the center of a primary eclipse is detectable at a level of $\sim 3 \mathrm{mCrab}$, so the ratio of the maximum non-eclipsed flux $(\sim 20 \mathrm{mCrab})$ to the minimum value at the mideclipse is about 6-7. The width of hard X-ray eclipse is found to be bigger than that in soft X-rays. The egress out of the hard X-ray eclipse is observed to be strongly variable, most probably due to absorption of the X-ray flux by accretion fbws and asymmetric, structured wind emanating from the supercritical accretion disk. Similar distortions of the eclipse egress was first observed by Ginga (18.4-27.6 keV) [15]. The hard X-ray spectrum (20-200 keV) does not noticeably change with the precession phase. All these facts suggest that the hard X-ray flux of SS433 is mostly generated in the hot extended corona formed in the central parts of the accretion disk. A detailed interpretation of the broad-band (3-90 keV) X-ray continuum of SS433 in terms of the multicomponent model including the accretion disk, jet and corona has been carried out by [16].

In the present paper we analyze hard X-ray eclipses of SS433 near the T3 moment as observed by INTEGRAL and interpret them in terms of our multicomponent geometrical model with account 
of the peculiar shape and strong variability of the primary eclipse.

\section{Precessional variability}

Dedicated INTEGRAL observations of SS433 were carried out in AO-1 for $500 \mathrm{ks}$, in AO-3 for $500 \mathrm{ks}$, in AO-4 in May 2007 for $466 \mathrm{ks}$, and in AO-5 for $900 \mathrm{ks}$. The AO-1 observations (6769 INTEGRAL orbits) were performed near the T3 moment (near the precessional phase 0 with maximum disk opening). Out of the eclipse, the source is reliably detected by IBIS/ISGRI up to 100 $\mathrm{keV}$, with the X-ray spectrum fitted by featureless power-law $d N / d t / d A / d E \sim E^{-2.8} \mathrm{ph} / \mathrm{s} / \mathrm{cm}^{2} / \mathrm{keV}$ [7, 8, 9], see Fig. 2. Considering a low significance of points above $\sim 100 \mathrm{keV}$, the exponential cut-off in the spectrum is still not excluded. The spectral continuum $2-100 \mathrm{keV}$ can be explained by thermal emission from cooling expanding plasma jet with temperature at the jet base $T_{0} \sim 22$ $\mathrm{keV}$, and a broad hot Comptonized region with $T_{c} \sim 20 \mathrm{keV}$ and optical depth $\tau \sim 0.2$ surrounding the thin X-ray jet [16].

In AO-2, SS433 fell within the FOV of IBIS when observing the Sagittarius Arm Tangent region, but no X-ray eclipses occurred during this program.

In AO-3, SS433 was observed around different precessional phases (INTEGRAL orbits 366369). One $X$-ray eclipse was partially measured again at the precessional phase close to zero, with an indication of much narrower eclipse or a sudden mid-eclipse (at $\psi_{o r b} \sim 1.03$ ) flux increase. Based on these data, the model for the source eclipse has been constructed in $[8,9]$; however, these data were not strongly constrained by precessional variability and allowed a broad range of the binary mass ratio $q \sim 0.1-0.5$.

In A0-4, the source was observed in May 2007; unfortunately, due to high variability, the source flux was very low, so we cannot include these data in the analysis of X-ray eclipses (see set II in Fig. 4). Note the strong increase of the eclipse width.

In AO-5, two consecutive eclipses of the source near the zero precessional phase were observed in October 2007. Adding all of these observations allowed the spectroscopy at different precessional phases (Fig. 2). However, the statistics is still insufficient to make orbital phaseresolved spectroscopy.

The combined AO1 - AO5 data (including those publically available) enabled us to measure, for the first time, the precessional hard X-ray variability (Fig. 1), which turned out to be quite significant and stable over several precessional periods. The maximum to minimum flux ratio of the precessional variability was found to be around 4, which is higher than in softer X-ray bands, and evidences for the hard X-ray emission originating closer to the basement of the visible part of the jets. The eclipsing and precessional variabilities, combined with spectroscopic data simultaneously taken with INTEGRAL observations by the 6-m telescope SAO RAS, were taken into account when attempting to model the light curve of the source by [8]. However, the results proved to be inconclusive. First of all, the spectral resolution of the optical observations (around 3000) was insufficient to definitely measure the radial velocity curve of the optical star, and the modeling of X-ray eclipse and precessional variability allowed fairly broad range of parameters, including the mass ratios $q \sim 0.1-0.3$. An independent spectral analysis of archival RXTE observations of SS433 [11] also suggests that it is hard to evaluate the mass ratio based on X-ray observations only. 


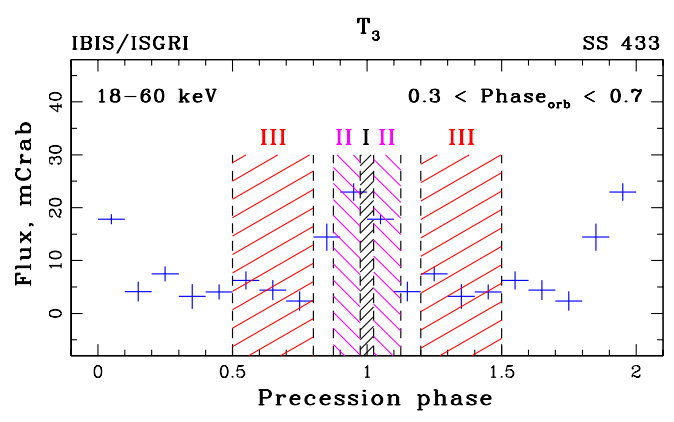

Figure 1: Precessional phase intervals chosen for spectral analysis of SS433.
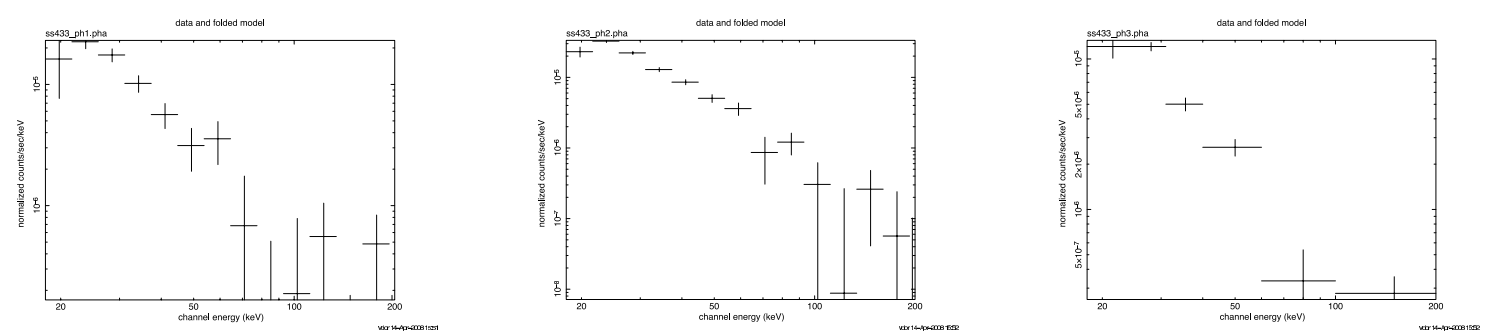

Figure 2: IBIS/ISGRI $10-200 \mathrm{keV} \mathrm{SS433} \mathrm{spectra} \mathrm{at} \mathrm{different} \mathrm{precessional} \mathrm{phases} \mathrm{(I,} \mathrm{II,} \mathrm{III} \mathrm{in} \mathrm{Fig.} \mathrm{1).} \mathrm{All}$ spectra can be fitted by a power-law model with the photon spectral index -2.8 .

\section{Hard X-ray spectra}

Our previous studies $[7,8,9]$ revealed that SS433 is a superaccreting galactic microquasar with hot corona above the accretion disk. SS433 is only one known massive X-ray binary in which such a corona is directly observed. To find spectral signatures of the hot corona, INTEGRAL observations (2003-2007) were separated into three segments in the precessional phase (Fig. 1) (limited by photon statistics). The resulted spectra are shown in Fig. 2, with no clear difference in the power-law spectral shape. This confirms the presence of a fairly broad region emitting in hard $\mathrm{X}$-rays with size compared to that of the accretion disk $\left(10^{11}-10^{12} \mathrm{~cm}\right)$, since (excluding orbital eclipses) the observed flux varies due to precession of the disk.

Guided by the constant shape of the hard X-ray spectrum, we summed up all precessional phases (to increase statistics). The obtained X-ray spectrum of SS433 in the 3-100 keV range can be fitted by two-component model (thermal X-ray emission from the jet and thermal comptonization spectrum from corona) elaborated in [16]. The model gives the best fit for $T_{c o r}=20 \mathrm{keV}$ with Thomson optical depth $\tau_{T}=0.2$ and mass outfbw rate in the jet $\dot{M}_{j}=3 \times 10^{19} \mathrm{~g} / \mathrm{s}$.

The best-fit corona parameters obtained from spectral fits suggest the electron density around $5 \times 10^{12} \mathrm{~cm}^{-3}$. Such a density is typical in the wind outfbwing with velocity $v \sim 3000 \mathrm{~km} / \mathrm{s}$ from a supercritical accretion disk with $\dot{M} \sim 10^{-4} \mathrm{M}_{\odot} / \mathrm{yr}$ at distances $\sim 10^{12} \mathrm{~cm}$ from the center, where a Compton-thick photosphere is formed [10, 20].

Note that despite several observations of primary eclipses by INTEGRAL, the observed hard $\mathrm{X}$-ray flux $\sim 5-20 \mathrm{mCrab}$ from the source is still insufficient for phase-resolved spectral analysis of an individual eclipse. Additional observations of SS433 by INTEGRAL are planned to allow 
phase-resolved spectroscopy of the primary eclipse.

\section{Primary eclipse and its peculiarity}

\subsection{INTEGRAL eclipses}

The INTEGRAL data were processed with both publically available ISDC software (OSA-7 version) and the original software elaborated by the IKI INTEGRAL team (for the IBIS/ISGRI telescope, see [18] for more detail).

In our analysis we have used data from our INTEGRAL observing program of SS433 plus publically available data of all observations where the source was in the FOV of the IBIS/ISGRI telescope ( $<13 \mathrm{deg})$. The exposure of selected data total an approximately 8.5 Ms. To perfume precessional phase-resolved analysis we ascribed to each SCW (Science Window or SCW, natural piece of INTEGRAL data - pointing observation with exposure $\sim 2-5 \mathrm{ks}$ ) appropriate orbital and precessional phases. The phases are calculated using the ephemeris provided by [10], and modified for INTEGRAL time data format. According to these ephemeris the relative orbital phase is:

$$
\Psi_{\text {orb }}=\left(T_{I J D}-1520.880\right) / 13.08211
$$

and the relative precessional phase is :

$$
\Psi_{\text {prec }}=\left(T_{I J D}-8037.03\right) / 162.375
$$

In Fig. 3 (left panels) we shown the exposure distribution of used observations with the phases. For clarity we presented two cycles. On the orbital phase panel "1" corresponds to the orbital minimum, and on the precessional phase panel " 1 " is the $T 3$ moment. While the exposure time for the orbital phases is distributed more or less homogeneously that in the precessional phase plane has excess near $T 3$ moment. The reason is that the main part of our SS433 INTEGRAL observing program were focused namely on observations of primary eclipses.

In this paper we focused on the precessional phase-resolved analysis. To exclude contamination of the orbital modulation on the results, in the present analysis we have used only observations with orbital phases from the range $0.3<\Psi_{\text {orb }}<0.7$. After this filtering we still have $\sim 2.8$ Ms of data distributed across all precession phases.

The dedicated INTEGRAL observations of primary eclipses of SS433 in 2003 and 2007 were obtained near the T3 precession phase when the accretion disk is maximally open. The data include:

I. INTEGRAL orbits 67-70 (May 2003), precession phase $\psi_{p r}=0.001-0.060$;

II. INTEGRAL orbits 555-556 (May 2007), precession phase $\psi_{p r}=0.980-0.014$;

III. INTEGRAL orbits 608-609 (October 2007), precession phase $\psi_{p r}=0.956-0.990$;

IV. INTEGRAL orbits 612-613 (October 2007), precession phase $\psi_{p r}=0.030-0.064$.

The phases are calculated using the ephemeris [10], according to which the orbital minimum is

$$
J D_{\text {MinI }}(\text { hel })=2450023.62+13.08211 * E,
$$

the $\mathrm{T} 3$ moment is

$$
J D_{T 3}=2443507.47+162.375 * E 1 .
$$

The observed eclipses are shown in Fig 4. In our analysis we have used only sets I, III, and IV and excluded set II (the one showing the most suppressed egress out of the primary eclipse). 

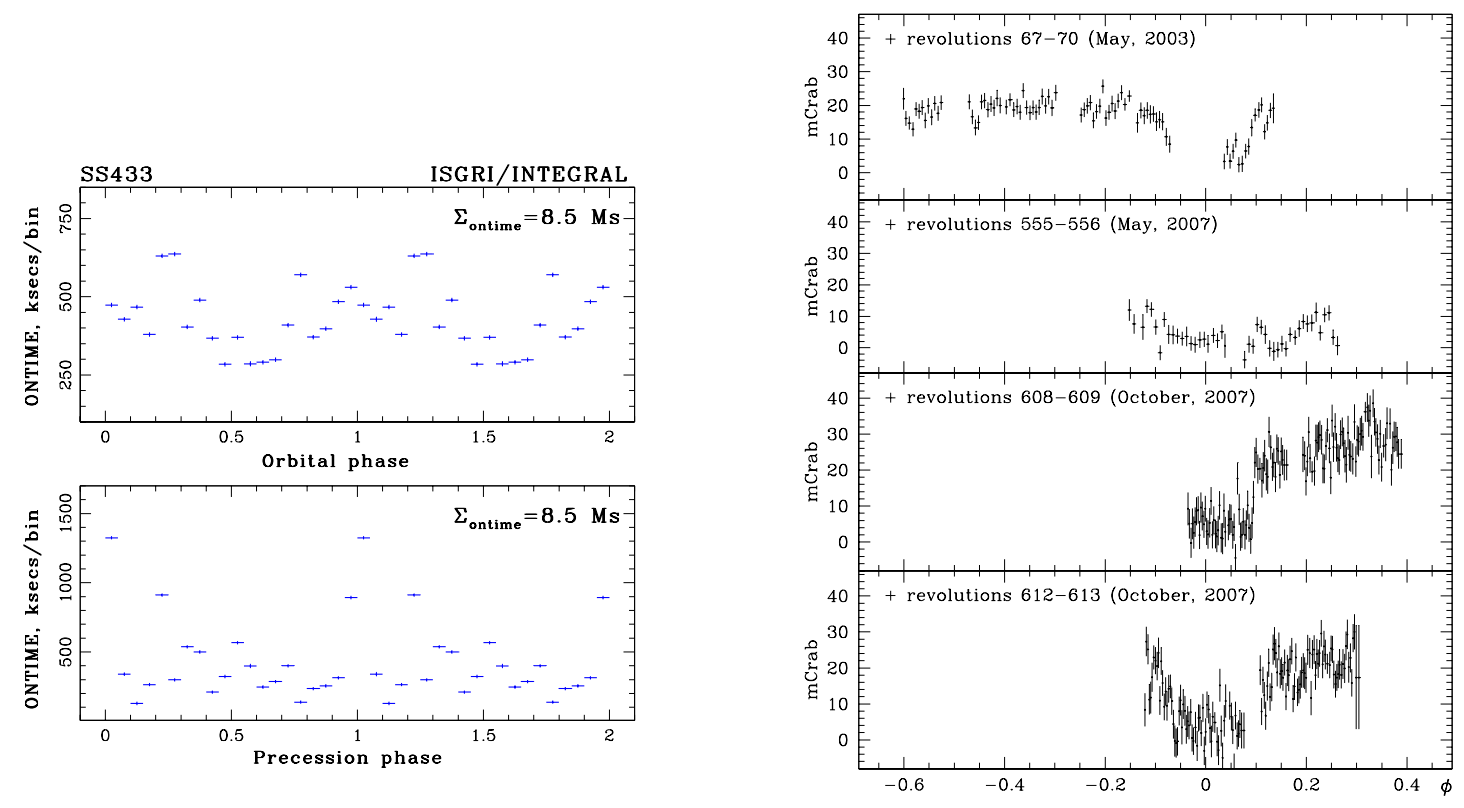

Figure 3: Distribution of the observing time from the orbital (upper) and the precession (bottom) phases.

Figure 4: The INTEGRAL primary eclipses of SS433 (IBIS/ISGRI data, 18-60 keV) I, II, III and IV sets (see the text).

\subsection{Variable shape of hard X-ray eclipse}

SS433 is known to be highly variable object at all wavelengths $[5,10,20,21]$. This is not surprising for a super critically accreting massive binary, considering various instabilities that should be immanently present in the mass outfbw from the optical star and in the accretion disk. It might appear that the primary eclipse of the hard X-ray emission coming from the regions close to the central black hole by the optical star should be stable. However, it is not the case for hard X-ray eclipse in SS433 (Fig. 4). As seen in this figure, the second eclipse observed in May 2007 has an unusually broad form: a shallow ingress to and broad egress from the eclipse, which may be due to the low level of non-eclipsed X-ray flux from the source $\sim 10 \mathrm{mCrab}$. Note that this particular eclipse is very similar to the one observed by Ginga [15]. However, already at the next T3 moment in October 2007 (sets III and IV in Fig. 4) the non-eclipsed X-ray flux was at a level of $20 \mathrm{mCrab}$ and the egress from eclipse restored its more familiar form (like in set I). This effect is particularly clearly visible on the combined X-ray eclipse light curve shown in Fig. 5. The reason for such a strong variability of the hard X-ray egress may be related to powerful inhomogeneous gaseous streams feeding the disk and the wind from the supercritical accretion disk. Indeed, the slow dense disk wind in SS433 should form a Compton-thick photosphere around the disk and funnels around relativistic jets [10]. The height of the funnel $\sim 10^{12}$, comparable to the accretion disk size, can be inferred from the observed $\sim 80$-s lag of the optical emission with respect to the X-rays from the jet base found in the simultaneous optical/X-ray observations of the source [20]. So it appears quite possible that the variable wind from the precessing supercritical accretion disk result in changes of the funnel structure, so even at the maximum opening disk phase (T3) the shape of hard X-ray 


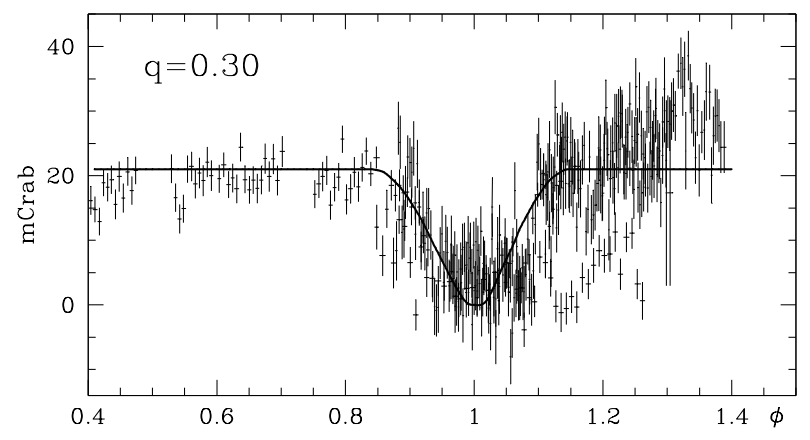

Figure 5: Combined INTEGRAL primary eclipses of SS433. The solid curve shows the model light curve for $q=0.3$ (see the text).
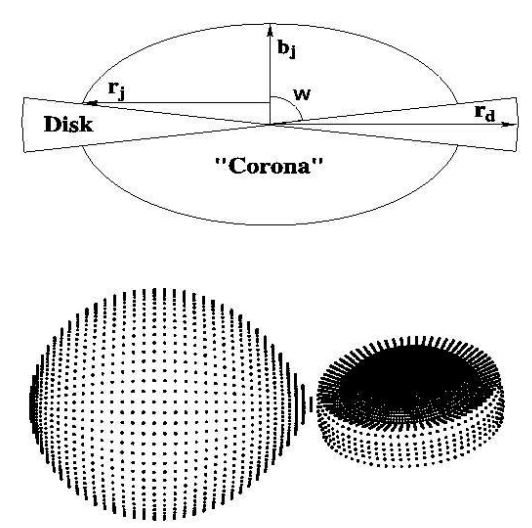

Figure 6: Geometrical model of the accretion disk and its "corona" (top) and the view of the SS433 geometrical model for $q=0.3$ at the maximum disk opening angle (bottom).

eclipse may appreciably vary depending on the current accretion rate through the disk. In this connection it is worth noticing the variable broad absorption-like feature that is seen immediately after the egress from the eclipse (at the orbital phases $\psi_{o r b} \sim 0.15-0.2$, see Fig. 4). It would be very interesting to confirm if this feature is due to the true absorption of X-ray emission or just refects the strong variability of the proper X-ray flux.

Therefore we conclude that the hard X-ray eclipse in SS433 is likely to be formed by both geometrical screening of the broad X-ray emitting region by the opaque star and by complex gaseous stream and/or inhomogeneous wind from the star and the supercritical accretion disk. This implies that to analyze the hard X-ray primary eclipse in SS433 by geometrical model we should use the most stable part of the observed eclipses, i.e. the upper envelope of the observed eclipse ingress. Moreover, in view of high variability, the use of only one X-ray eclipse for analysis can lead to erroneous determination of the binary system parameters.

\subsection{Geometrical model of SS433}

To analyze hard X-ray eclipses of SS433 we used a geometrical model developed earlier to the interpretation of the Ginga data [1] and the INTEGRAL light curve [8]. We consider a close binary system consisting of an (opaque) "normal" star limited by the Roche equipotential surface and a relativistic object surrounded by an optically and geometrically thick "accretion disk". Relativistic jets are directed perpendicularly to the disk plane. The "accretion disk" includes the disk itself and an extended photosphere formed by the outfbwing wind. The orbit is circular, the axial rotation of the normal star is assumed to be synchronized with the orbital revolution.

The disk and "jets" are precessing in space and change the orientation relative to the normal (donor) star. The disk is inclined with respect to the orbital plane by the angle $\theta$. A cone-like funnel is located inside the disk and is characterized by the half-opening angle $\omega$, thus the opaque disk 
body (see Fig. 6) is described by the radius $r_{d}$ and the angle $\omega$. The central object is surrounded by a transparent homogeneously emitting spheroid with a visible radius $r_{j}$ and height $b_{j}$ which could be interpreted as a "corona" or a "thick jet" (without any relativistic motion). Here $r_{j}, b_{j}$ and $r_{d}$ are dimensionless values expressed in units of the binary separation $a$. The radius of the normal star is determined by the relative Roche lobe size, i.e. by the mass ratio $q=m_{x} / m_{v}\left(m_{x}\right.$ is the mass of the relativistic object).

Only the "corona" is assumed to emit in the hard X-ray band, while the star and disk eclipse it in the course of the orbital and precessional motion. During precession the inclination of the disk with respect to the observer changes, causing different visibility conditions for the "corona". The "corona" is partially screened by the cone disk edge, which can explain qualitatively the change of the non-eclipsed X-ray flux with precession phase. Observations of precessional variability can thus be used to obtain a "vertical" scan of the emitting structure, restricting the parameters $b_{j}$ and $\omega$. The orbital (eclipse) variability observations scan the emitting structure "horizontally", restricting possible values of $r_{d}, \omega, q$ and $r_{j}$. The joint analysis of the precessional and eclipse variability enables us to reconstruct the spatial structure of the region in the accretion disk center where the hard X-rays are produced and to estimate the binary mass ratio $q$.

The position of the components of the system relative to the observer is determined by the binary orbit inclination angle $i=78.8^{\circ}$, the disk inclination angle to the orbital plane $\theta=20.3^{\circ}$, and the precessional phase $\psi_{p r}$. Phase $\psi_{p r}=0$ corresponds to the maximum disk opening of SS433 (T3 moment, maximum separation between the moving emission lines) and $\psi_{p r}=0.34,0.66$ when the disk is seen edge-on (at the moving emission line crossover moments $\mathrm{T} 1$ and $\mathrm{T} 2$, respectively).

\subsection{Light curves analysis}

Free parameters of our model for the orbital and precessional variability of SS433 include: the binary mass ratio $q=m_{x} / m_{v}$ determining the relative size of the normal star, the disk parameters $r_{d}, \omega$ determining the form of the disk, the thick "jet" or "corona" parameters $r_{j}, b_{j}$ determining the form of the hot corona. Note that the X-ray emitting region is qualitatively different for a thin narrow "jet" with $r_{j} \ll b_{j} \sim a$ and a thin short "jet" with $r_{j} \ll b_{j} \ll a$. If $r_{j}>b_{j}$, it is more appropriate to term it as a corona (or a thick "jet"). For each value of $q$ from the range $0.05-1.0$, we found other parameters best-fitting simultaneously the orbital and precession light curves. The precession variability amplitude was assumed to be $A_{p r} \simeq 5-7$. The $\chi^{2}$ criterion was used to evaluate the goodness of fits.

To examine as fully as possible all system parameters, we have used different variants of the observational data modeling. These included:

1) the analysis of light curves consisting of individual points;

2) the analysis of average light curves;

3) the analysis of individual observational sets (I, III, and IV);

4) the analysis of the combined data from sets I, III and IV;

5) the analysis of the entire eclipse, including the ingress and egress phases;

6) the analysis of only ingress to the eclipse (orbital phases $\psi_{\text {orb }}=0.8-1.0$ ).

The last analysis appears to be the most reliable, since all observations of the primary eclipse of SS433 evidence that the form of egress out of eclipse is strongly variable and thus cannot be described by purely geometrical model of eclipses. 
Our numerous calculations led to the following conclusions.

1) The results obtained using individual and average light curves are identical (i.e. the using of the average light curve does not add errors to the obtained parameters). This might seem to be obvious, but we directly checked it.

2) The results obtained from the analysis of individual data sets I, III, IV are the same as ones obtained from the analysis of the combined data.

Taking into account the above conclusions, below we shall show the model light curve for the combined data sets I, III and IV.

3) When searching for the best-fit model parameters at different $q$, we found that solutions obtained for the entire primary eclipse and for the eclipse ingress only are not very different. However, taking into account highly variable character of the eclipse egress noted above, below we shall discuss solutions and model parameters obtained from the analysis of the eclipse ingress part only.

4) In our previous analysis of the primary orbital eclipse observed by INTEGRAL [8] we found that for all models the best-fit values (corresponding to the minimum $\chi^{2}$ ) are obtained for a maximum possible radius of the disk $r_{d}$ and a maximum possible value of the angle $\omega$. This conclusion is confirmed here using new data. We also confirm that parameters $b_{j}$ and $\omega$ are correlated.

5) The minimum deviation of the best-fit model eclipsing light curve from observed points is reached for the small mass ratio $q \sim 0.1$ and a long X-ray emitting "jet" $\left(b_{j}>0.5\right)$ with the base radius varying in the wide range $\left(r_{j}=0.05-0.25\right)$. This means that the "jet" can be long but either thin or thick. However, the main objection to model of the long "jet" comes from the impossibility to describe the observed precessional variability. This is illustrated in Fig. 7. We conclude that at small mass ratio $q \leq 0.2$ our model does not simultaneously fit both orbital and precessional variability of SS433 observed by INTEGRAL. The precessional light curve could be fitted at the small mass ratio by a short X-ray emitting "jet", but then the flux at the center of the primary eclipse would be zero, again in contradiction to observations - in all cases, INTEGRAL detects a non-zero $\mathrm{X}$-ray flux of $\sim 3 \mathrm{mCrab}$ at the middle of hard X-ray eclipse (Fig. 4).

6). For $q=0.3-0.5$ our model provides simultaneously good fits to the orbital and precessional variability of SS433, although $\chi^{2}$ deviations are formally worse than for small values of $q$. The deviations increase with $q$, so the minimal $\chi^{2}$ are obtained for $q=0.3$. The model parameters correspond to an extended corona comparable to the accretion disk in width $\left(r_{j} \sim r_{d}\right)$ and with small vertical size $\left(b_{j} \sim 0.15-0.20\right)$. This geometry of the hard X-ray emitting region is caused by the wide primary eclipse minimum and large amplitude of the precessional variability. So the precessional light curve is shaped by the outer parts of the disk eclipsing the high-temperature corona. Fig. 8 shows the orbital and precessional light curves for the best-fit binary mass ratio $q=0.3$. Figs. 7 and 8 clearly demonstrate the change in the primary eclipse minimum width for different binary mass ratios.

\subsection{Masses of the components}

From the analysis of the primary eclipse at the precessional phase $\Psi=0.1$ (the upper envelope) with account for precessional variability we have obtained the mass ratio $q=m_{x} / m_{v} \simeq 0.3$. Using the mass function of the optical component of SS433 derived from spectroscopic observations by 

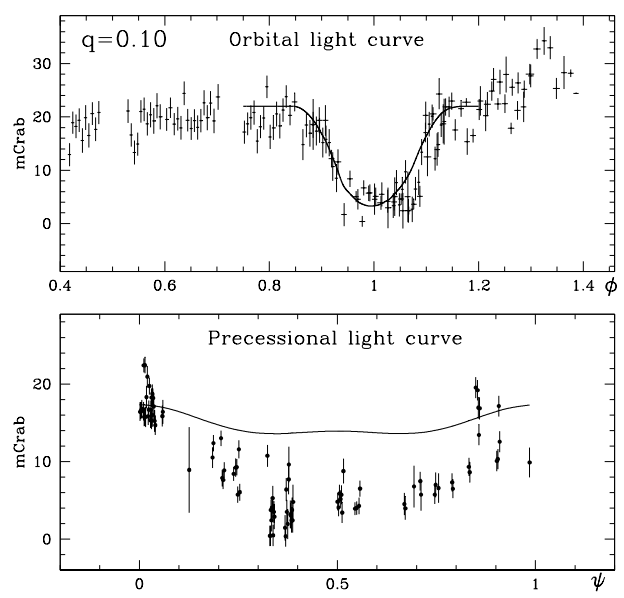

Figure 7: Orbital (top) and precessional (bottom) light curves of SS433 from INTEGRAL observations for model with $q=0.1$. The long jet model is shown (see the text). The model can not simultaneously fit the eclipse and precessional light curves.

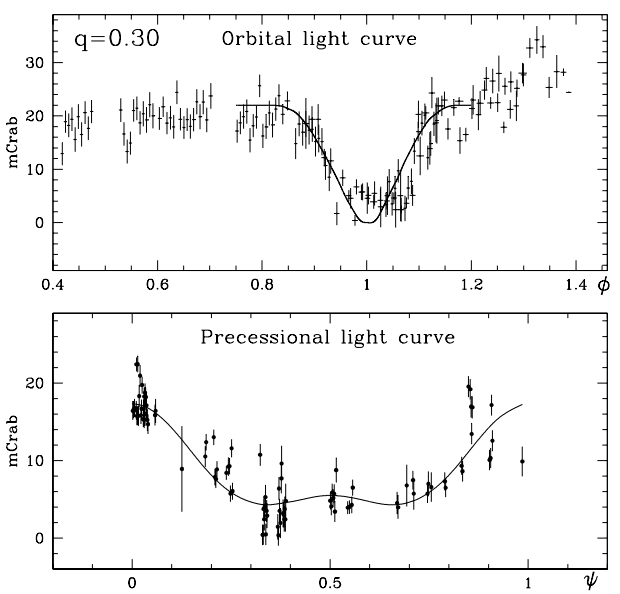

Figure 8: $\mathrm{X}$-ray eclipse and precessional light curve of SS433 for the mass ratio $q=0.3$ Note highly variable eclipse egress. The solid curve shows the model eclipsing and precessional light curve (see the text).

[14],

$$
f_{v}(m)=\frac{m_{x} \sin ^{3} i}{(1+1 / q)^{2}} \simeq 0.268 M_{\odot},
$$

we get masses of the relativistic object and the optical star:

$$
m_{x} \simeq 5 M_{\odot}, \quad m_{v} \simeq 15 M_{\odot} .
$$

The high mass value of the relativistic object in SS433 strongly suggests its being a black hole.

\section{Discussion}

Our analysis confirms the nature of SS433 as a superaccreting microquasar with black hole. The obtained high value of the binary mass ratio in SS433 $q=0.3$ allows us to easily explain a substantial amplitude $\left(\sim 0^{m} .5\right)$ of the optical variability at the minimum of eclipse with precessional phase. The orbital inclination of SS433 is known from independent analysis of moving emission lines ( $i \simeq 78^{\circ} .8$ ), so at small $q \simeq 0.1 \div 0.2$ the small relative radius of the Roche lobe of the relativistic object should have caused the total eclipse of the bright precessing accretion disc around the relativistic object by the optical A7I-star, implying a constant optical flux at the center of the eclipse at different precession phases. This contradicts to observations (see e.g. [13]). At $q=0.3$ the size of the Roche lobe of the relativistic object is relatively large to cause partial primary optical eclipse of the accretion disc by the A7I-star at all precession phases, implying significant precessional variability of the minimum brightness at the middle of the primary eclipse. 
A small value of the binary mass ratio was found by [15] from the analysis of soft $(k T \simeq$ $1 \div 10 \mathrm{keV}$ ) X-ray eclipses of SS433. As follows from the results of modeling of broad-band X-ray spectrum of SS433 [16], in this energy range thermal emission of relativistic jets with temperature decreasing along the jet dominates. The observed broad width of soft X-ray eclipse was interpreted by the authors [15], by applying the purely geometrical model of the eclipse of jets by the optical star with sharp limb, found the small value of the binary mass ratio $q=0.15$. The observed independence of the soft X-ray eclipse width on energy was the main argument justifying this model and low mass ratio. However, this argument holds only if the temperature along jets does not change with distance from the relativistic object. In fact it decreases along jets (unless some additional heating mechanism is assumed), so at energies $\sim 10 \mathrm{keV}$ mainly central parts of the jets are eclipsed. Consequently, the duration of purely geometrical eclipses must be longer at $10 \mathrm{keV}$ than at $\sim 1 \mathrm{keV}$ where cooler periphery of the jets is eclipsed [11]. The independence of the X-ray eclipse duration on energy of X-ray photons in the 1-10 keV range can be due to the compensation of the effect of decreasing temperature along jets and the increase of soft X-ray absorption in the extended atmosphere of the optical star. So the effective radius of the eclipsing star increases at smaller energy of X-ray photons which are mostly emitted at smaller temperature along the jets. This explains the constant width of X-ray eclipse in the 1-10 keV energy range. Turning this argument around, from the independent width of the X-ray eclipse in this energy range we can conclude that the eclipsing A7I-star in SS433 has not a sharp limb, so that X-ray eclipses in SS433 are not purely geometrical and are suffered from extinction in the stellar wind from the optical star. This lends additional support to our interpretation of X-ray eclipses in SS433 which makes use of only the upper envelope of variable eclipsing hard X-ray light curve.

\section{Conclusion}

The discovery of highly variable hard X-ray eclipse at precessional phases corresponding to the maximum disk opening angle for the observer and significant precessional variability are the main findings of our INTEGRAL observations of SS433. It is shown that the duration of the primary Xray eclipse in different epochs changes by $\sim 2$ times. This implies that X-ray eclipses in SS433 are not purely geometrical, they are shaped by absorption in a powerful stellar wind and gas streams from the optical star. So to infer the binary mass ratio $q$ from the analysis of X-ray eclipses we have used only the upper envelope of the eclipsing light curve. The analysis of this upper envelope in combination with the precessional light curve yielded the mass ratio estimate $q=0.3$. The relatively high value of the binary mass ratio provides an easy explanation of peculiarities of the optical variability of SS433, in particular, the substantial precessional variability of the minimum brightness at the middle of the primary optical eclipse.

Using the mass function of the optical star found by [14] $f_{v}(m)=0.268 M_{\odot}$ and the value of $q=m_{x} / m_{v} \simeq 0.3$ inferred from our analysis, we concluded that the masses of binary components of SS433 are $m_{x} \simeq 5 M_{\odot}, m_{v} \simeq 15 M_{\odot}$. The high mass of the relativistic object leaves no doubts that it is a black hole.

The independence of hard X-ray spectrum on the accretion disk precession phase suggests that hard X-ray emission $(k T=20-100 \mathrm{keV})$ is formed in an extended, hot, quasi-isothermal corona. 
The heating of the corona can be due to transformation of kinetic energy of relativistic jets to an inhomogeneous wind outfbw from the precessing supercritical accretion disk [3].

The Monte-Carlo simulations of broadband X-ray spectrum of SS433 at the maximum disk opening precessional phases [16] allowed us to determine the main physical characteristics of the hot corona (temperature $T_{\text {cor }} \simeq 20 \mathrm{keV}$, Thomson optical depth $\tau \simeq 0.2$ ), as well as to estimate the mass outflbw rate in jets $\dot{M}_{j}=3 \times 10^{19} \mathrm{~g} \mathrm{~s}^{-1}$ yielding the kinetic power of the jets $\sim 10^{39} \mathrm{erg} \mathrm{s}^{-1}$.

\section{Acknowledgments}

The authors thank M. Revnivtsev for useful discussions. The work is partially supported by the RFBR grants 07-02-00961 and 08-02-01220.

\section{References}

[1] E.A. Antokhina, E.V. Seifina, A.M. Cherepashchuk, SvA 36143 (1987).

[2] E.A. Antokhina, A.M. Cherepashchuk, SvA 31295 (1987).

[3] M.C. Begelman, A.R. King, J.E. Pringle, MNRAS 370399 (2006).

[4] A.M. Cherepashchuk, MNRAS 194761 (1981).

[5] A.M. Cherepashchuk, Sov. Sci. Rev. Ap. Space Phys. (Ed. by R.A.Sunyaev) 71 (1988).

[6] A.M. Cherepashchuk et al, ApSS 22933 (1995).

[7] A.M. Cherepashchuk et al, $A A 411441$ (2003).

[8] A.M. Cherepashchuk et al, $A A 437561$ (2005).

[9] A.M. Cherepashchuk et al, Proc. 6th INTEGRAL Workshop, ESA SP-622, p. 319 (2007).

[10] S.N. Fabrika, Astrophys. Space Phys. Rev. 121 (2004).

[11] E.V. Filippova et al., $A A 460125$ (2006).

[12] D.R. Gies, W. Huang, M.V. McSwain, ApJ 578 L67 (2002)

[13] V.P. Goranskij, V.F. Esipov, A.M. Cherepashchuk, Astron. Rep. 42, 209; 336 (1998).

[14] T.C. Hillwig, D.R. Gies, ApJ 676 L37 (2008).

[15] N. Kawai, M. Matsuoka, H.-C. Pan, G.C. Stewart, PASJ 41491 (1989).

[16] Yu. Krivosheev et al., MNRAS (submitted) (2008).

[17] B. Margon, ARAA 22507 (1984).

[18] S. Molkov et al., Astron. Lett. 30534 (2004).

[19] M. Namiki et al., PASJ 55281 (2003).

[20] M. Revnivtsev et al., AA 424 L5 (2004).

[21] M. Revnivtsev et al., AA 447545 (2006).

[22] N.I. Shakura, R.A. Sunyaev, AA 24337 (1973). 